\title{
An Investigation of Long and Short Time-Constant Persistent Photoconductivity in Undoped GaN Grown By RF-Plasma Assisted Molecular Beam Epitaxy
}

A.J. Ptak, V.A. Stoica, L.J. Holbert, M. Moldovan and T.H. Myers

Department of Physics, West Virginia University, Morgantown, WV 26506

\begin{abstract}
Photoconductance decay and spectral photoconductance measurements were made on a set of ten undoped layers of GaN grown by rf-plasma MBE. The layers, also characterized by Hall, photoluminescence and reflectance measurements, represented a wide variety in electrical and optical properties, and several were grown under atomic hydrogen. Spectral photoconductance indicated transitions at 1.0-1.1, 1.92, 2.15, 3.08 and 3.2-3.4 eV. All layers exhibited persistent photoconductivity to some degree. In contrast with previous reports, a clear correlation was not observed between persistent photoconductivity and yellow luminescence or, indeed, with any measurement made. Analysis of photoconductance decay indicates that more than one type of persistent photoconductivity may be present.
\end{abstract}

\section{INTRODUCTION}

Persistent photoconductivity (PPC) has been observed in n-type, p-type and undoped $\mathrm{GaN}$ grown by molecular beam epitaxy (MBE) $[1,2,3,4]$ and metal organic chemical vapor epitaxy (MOCVD). [5,6,7] Interestingly, it has not yet been seen in cubic GaN. [8] The almost universal presence of PPC may indicate that it is tied to native defects. The successes that have been obtained with $\mathrm{GaN}$ light emitters indicate PPC is not a problem for such structures, but this ubiquitous phenomenon will definitely affect the performance of ultraviolet detectors, X-ray detectors and field effect transistors. All these structures rely on fabricating insulating, preferably undoped, layers. Thus, PPC in undoped layers must be understood and, if possible, controlled. We report on a study of photoconductivity effects in ten samples grown by rfplasma MBE which exhibit a wide range of optical and electrical properties.

\section{EXPERIMENTAL DETAILS}

The GaN layers investigated in this study were selected from the large number of samples grown at West Virginia University over the last several years. Growth procedures are reported elsewhere. [9] In brief, all samples were grown under Ga-rich conditions in the range 675 to $750^{\circ} \mathrm{C}$, with four samples grown under an atomic hydrogen flux. The samples were chosen to reflect a wide range in

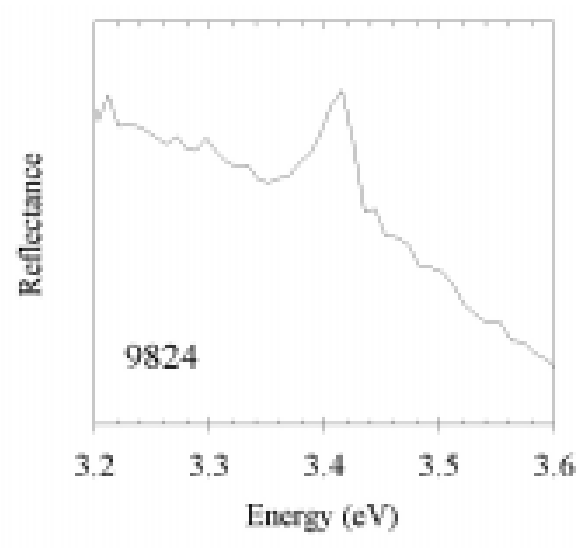

Figure 1. Reflectance spectrum of sample 9824 with a free exciton peak. 
Table 1. Various properties of the ten samples included in this investigation. See text for details of each measurement.

\begin{tabular}{|c|c|c|c|c|c|c|c|}
\hline $\begin{array}{c}\text { Sample } \\
(\text { Thickness }) \\
(\mu \mathrm{m})\end{array}$ & $\begin{array}{c}\tau_{50 \%} \\
\tau_{10 \%} \\
(\mathrm{~s})\end{array}$ & $\begin{array}{c}\tau_{\text {short }} \\
(\mathrm{ms})\end{array}$ & $\begin{array}{c}\text { a-H } \\
\text { during } \\
\text { growth }\end{array}$ & $\begin{array}{c}\text { Yellow } \\
\mathrm{PL}\end{array}$ & $\begin{array}{c}\text { Mobility } \\
\left(\mathrm{cm}^{2} / \mathrm{Vs}\right)\end{array}$ & $\begin{array}{c}\mathrm{n} \\
\left(10^{16}\right. \\
\left.\mathrm{cm}^{-3}\right)\end{array}$ & $\begin{array}{c}\text { Exciton } \\
\text { in RT } \\
\text { Refl. }\end{array}$ \\
\hline $\begin{array}{c}9824 \\
(9.2)\end{array}$ & $\begin{array}{c}4.0 \\
246\end{array}$ & 0.7 & & weak & 202 & 1.7 & $\begin{array}{c}\text { yes } \\
\text { strong }\end{array}$ \\
\hline $\begin{array}{c}9821 \\
(2.0)\end{array}$ & $\begin{array}{c}4.8 \\
622\end{array}$ & 0.5 & & weak & 15 & 9.2 & no \\
\hline $\begin{array}{c}9826 \\
(7.5)\end{array}$ & $\begin{array}{c}5.1 \\
4,044\end{array}$ & 1.2 & & weak & 9.0 & 1.8 & yes \\
\hline $\begin{array}{c}9825 \\
(2.6)\end{array}$ & $\begin{array}{c}7.0 \\
7,171\end{array}$ & 1.4 & & not det. & 12 & 1.7 & yes \\
\hline $\begin{array}{c}9910 \\
(1.72)\end{array}$ & $\begin{array}{c}13 \\
29,521\end{array}$ & 1.2 & weak & 330 & 0.10 & yes \\
\hline $\begin{array}{c}9671 \\
(1.0)\end{array}$ & $\begin{array}{c}64 \\
6,704\end{array}$ & 2.3 & yes & mod. & 120 & 77 & no \\
\hline $\begin{array}{c}9730 \\
(0.98)\end{array}$ & $\begin{array}{c}214 \\
21,061\end{array}$ & 2.3 & yes & not det. & 4 & 7.2 & no \\
\hline $\begin{array}{c}9710 \\
(0.85)\end{array}$ & $\begin{array}{c}293 \\
32,564\end{array}$ & 1.8 & yes & weak & 50 & 85 & no \\
\hline $\begin{array}{c}9823 \\
(2.1)\end{array}$ & $\begin{array}{c}308 \\
8,703\end{array}$ & 1.6 & yes & weak & 232 & 140 & yes \\
\hline $\begin{array}{c}9822 \\
(2.1)\end{array}$ & $\begin{array}{c}357 \\
52,922\end{array}$ & 1.0 & & mod. & 13 & 0.67 & $(?)$ \\
\hline
\end{tabular}

electrical properties, as indicated in Table 1. Several of the samples were of high electrical and structural quality, as indicated by their high mobility and low carrier concentration. Samples 9824 and 9826 exhibited free excitonic photoluminescence (PL) at low temperature. Room temperature reflectance measurements, such as that shown in Figure 1, also indicated the presence of free excitons in about half the samples. Fitting this peak with a Lorentzian function appropriate to excitonic transitions indicates a transition energy of $3.412 \mathrm{eV}$, which is comparable to the value of $3.411 \mathrm{eV}$ reported recently by Viswanath et al. [10] for the free exciton (A) transition at room temperature. This indicates a bandgap of 3.438-3.400 eV at room temperature based on reported values of the exciton binding energy.[10]

\section{PHOTOCONDUCTANCE DECAY MEASUREMENTS}

Persistent photoconductivity was measured by monitoring sample resistance with a Keithley 175A multimeter after exposure to light. Samples were stored in the dark for approximately three to four days prior to light exposure in order to determine a baseline dark resistance, followed by exposure to either a bright white light source with the infrared removed via filtering, or to a weak light source consisting of filtered light corresponding to the yellow PL peak position. Either source of light led to a PPC effect, similar to that shown in Figure 2. The intensity varied, but the temporal decay was similar. Many authors $[1,4,6,11]$ have described PPC in GaN using a so-called "stretched exponential" 


$$
\mathrm{C}(\mathrm{t})=\mathrm{C}(0) \exp \left[\left(\mathrm{t} / \tau_{\mathrm{ppc}}\right)^{\beta}\right] \quad(1)
$$

The two fits shown in Figure 2 represent this function. By better fitting the shorter time response, we obtained a value of $\beta$ of about 0.2 , comparable to that reported in the studies by Li et al. [4] and Beadie et al. [6] By better fitting the longer time response, we obtained a value for $\beta$ closer to 0.26 , similar to that found in the studies by Qiu et al.[1] and Chen et al. [11]. Since there is ambiguity in interpreting our results in terms of a stretched exponential, we instead report two values for each sample, the time taken for the PPC to decay to $50 \%\left(\tau_{50 \%}\right)$ and to $10 \%\left(\tau_{10 \%}\right)$ of its maximum value. The data in Table 1 have been sorted by $\tau_{50 \%}$. Note that the two decay times do not appear to be tightly coupled.

In addition to "standard" PPC measurements, we also investigated the shorter timescale response of our samples by measuring the frequency response of the photoconductance signal to chopped radiation. Either below band gap (filtered) or unfiltered light from a strong white-light source was used. Except for changes in initial signal strength, both resulted in essentially identical decay curves. The observed decay was found to follow the classic response function associated with an exponential decay,

$$
\mathrm{s}(\mathrm{f})=\mathrm{s}(0) /\left[1+\left(2 \pi \mathrm{f} \tau_{\text {short }}\right)^{2}\right]^{1 / 2},
$$

from which the PC response time $\tau_{\text {short }}$ was determined. There was always a "residual" $\mathrm{PC}$ signal at the highest frequency measured which is likely related to the true intrinsic $\mathrm{PC}$ response. Representative data is shown in Figure 3. Examination of the results listed in Table 1 indicate that $\tau_{\text {short }}$ appears to be correlated with the longer time constant PPC signal for the samples with smaller PPC effects, but essentially is decoupled from this phenomenon for samples with more significant PPC. We believe that this indicates at least two mechanisms for PPC: the more publicized very long time-constant effect and a second one many orders of magnitude shorter. As discussed below, it is conceivable that both are due to the same native defect.

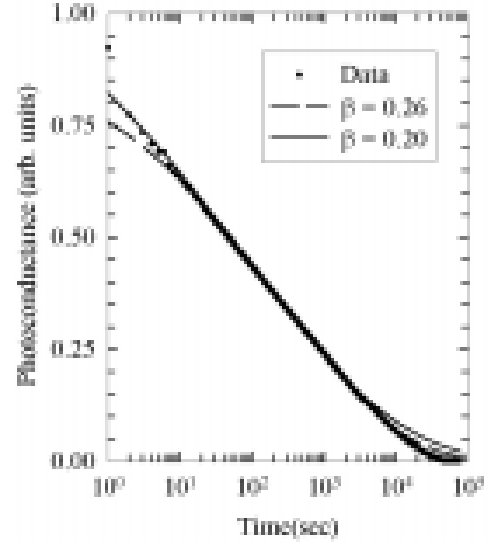

Figure 2. PPC and stretched exponential fit for 9671 .

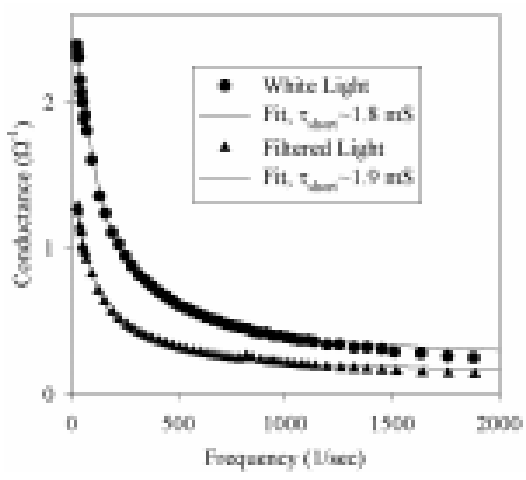

Figure 3. Frequency response of photoconductivity signal in sample 9710 for unfiltered white light and light filtered to correspond to the yellow PL peak. 


\section{PPC AND \\ PHOTOLUMINESCENCE}

Room temperature photoluminescence measurements indicated two primary features: a free excitonic peak at about 3.415-3.420 eV (accompanied by a phonon replica at $3.36 \mathrm{eV}$ ) and the so-called "yellow" luminescence band which shifts from sample to sample but is centered between 1.9 to $2.2 \mathrm{eV}$, as illustrated in Figure 4. The yellow PL for all the samples was relatively weak, and not detectable for two of the samples investigated. Two observations can be made relating PPC and yellow PL. First, as noted in Table 1, the two samples without detectable yellow PL (9825 and 9710) still exhibited significant PPC effects. Second, the magnitude of the yellow PL in the samples indicated as having a weak signal was comparable, yet this sample set exhibited a wide range in PPC effects. The sample with the strongest PPC effect (9822) did have about three to four times the yellow PL than the sample exhibiting the least PPC (9824), but it also exhibited a peak at about $3.2 \mathrm{eV}$ that could be indicative of cubic inclusions.

While other studies [8] have indicated a correlation between PPC and yellow PL, our results are in agreement with at least one other study [6] that find PPC is not directly correlated with PL features. It may be only that one of the transitions resulting in yellow $\mathrm{PL}$ is related to the origin of PPC, with the other related to additional impurities or defects. Thus, the absence of yellow PL does not indicate that the sample is free from PPC. In addition, the strength of yellow PL is not a direct indication of the magnitude of the PPC effect. The observation, both here and by others [1], that PPC is generated for photon energies above $1.1 \mathrm{eV}$ is likely indicative of the fact that electron-hole pairs generated by any light absorption process, such as the states indicated below by spectral PC, can generate PPC in undoped GaN. We personally favor the proposal put forth by Qiu and Pankove [1] that PPC behavior in n-type and undoped GaN is due to the presence of hole traps, with the Ga vacancy a likely candidate. Indeed, a recent study [12] of room-temperature PL presents a convincing argument that a state at about $1.1 \mathrm{eV}$ is due to the Ga vacancy, which can be coupled with photocapacitance measurements [14] indicating a persistent level at about this same energy. The Ga vacancy is a tripleacceptor, and has been predicted to occur at about this energy. [13] As such, it is conceivable that it could trap holes with different release times, resulting in both the faster and slower PPC time regimes we have observed.

\section{SPECTRAL PHOTOCONDUCTIVITY}

Spectral photoconductivity measurements were also made on the samples. A weak probe beam was used whose intensity was kept low enough so that the background current was not significantly altered. The signal was detected using a lock-in amplifier with the probe beam modulated at frequency of 4 to $5 \mathrm{~Hz}$ to maximize signal response. Spectra were corrected for variations in incident photon flux. Within the resolution of signal to noise, there were no significant differences between the spectra. The results for 
the six spectra with the best signal to noise are shown in Figure 5. The signal was not scaled by sample thickness. Samples 9826 and 9824 were significantly thicker than the others (7.5 and $9.2 \mu \mathrm{m}$ vs. 1 to $2 \mu \mathrm{m}$, as listed in Table 1) resulting in a stronger belowgap signal which allows better determination of changes in the PC signal.

An initial strong onset of PC was observed at or below $1.1 \mathrm{eV}$. Such behavior has been observed previously in spectral PC, [1] although for p-type material. In contrast, the only other spectral PC investigations we found in the literature indicated PC which increased exponentially for below band gap illumination in nominally undoped n-type layers. [2,3] Our result correlates with the observation that yellow PL can be excited by photons in the spectral range of $1.1 \mathrm{eV}$ to above bandgap energies. This $1.0-1.1 \mathrm{eV}$ level is comparable to one observed in photocapacitance measurements. [14] Measurements made on the thinner samples also indicated a clear transition at $3.08 \mathrm{eV}$ and a signal indicative of the onset of strong free excitonic absorption, as indicated in the inset in Figure 5. The effect of absorption below the free exciton energy is magnified for the two thicker samples. Photocurrent onset features were indicated at $1.92 \mathrm{eV}$ and $2.15 \mathrm{eV}$ in addition to the $3.08 \mathrm{eV}$ feature. As shown in the inset, one of the thick samples, 9826, also indicated a PC onset at $3.26 \mathrm{eV}$, while both of the thicker samples exhibited a strong resonance peak centered around $3.395 \mathrm{eV}$.

A recent PL study has proposed that yellow luminescence is related to an electronic transition from a shallow donor to a level at $1.08 \mathrm{eV}$, therein attributed to the $\mathrm{Ga}-$ vacancy.[12] This gives a room temperature peak of about $2.3 \mathrm{eV}$ for yellow PL for typical shallow donors. Analysis of variable temperature Hall measurements on the samples in this study indicated a donor activation energy of $\sim 180 \mathrm{meV}$. The onset observed at $3.26 \mathrm{eV}$ in the thickest sample could be interpreted as electron excitation from the valence band to this level. This is not a strong transition since this level would be strongly populated at room temperature. Using $3.438 \mathrm{eV} \mathrm{[10]} \mathrm{as} \mathrm{the} \mathrm{band} \mathrm{gap} \mathrm{energy}$ and $180 \mathrm{meV}$ as the donor, transitions with a state at $1.08 \mathrm{eV}$ would predict yellow PL

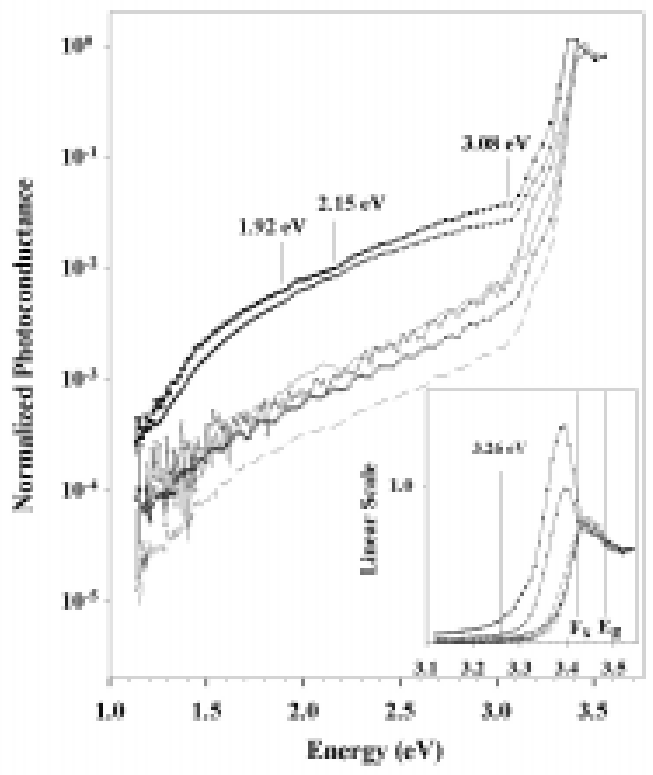

Figure 5. Spectral photoconductance measurements showing photocurrent onset features. The inset shows a higher resolution measurement for the excitonic region. 
peaked at $2.18 \mathrm{eV}$, close to that observed. This also correlates with the $2.15 \mathrm{eV}$ onset observed in spectral PC. If the level at $1.08 \mathrm{eV}$ is due to a Ga vacancy, calculations [15] indicate that it can be split by hydrogenation. A residual background of hydrogen is often observed in our layers. Thus, the possibility exists that the PC onset at $1.90 \mathrm{eV}$ is related to splitting of the Ga-vacancy level, and would explain in part the broad nature of this PL band. The relative contributions of the two bands could also account for the shift observed for this peak from sample to sample. The splitting observed in spectral PC, $0.25 \mathrm{eV}$, is close to that predicted. [15] At this point, though, this is quite speculative. Finally, low temperature PL of most of these samples featured donor-acceptor recombination indicative of an unidentified acceptor level at about $210 \mathrm{meV}$ (assuming shallow donors at $\sim 30 \mathrm{meV}$ ). Transitions from this level to the donor level at $180 \mathrm{meV}$ would predict a transition energy of $3.05 \mathrm{eV}$, close to that observed in spectral PC. The peak at $3.395 \mathrm{eV}$ in the spectral PC could be due to an unidentified residual donor that has been emptied by the acceptor, thus not contributing to the carrier concentration.

\section{CONCLUSIONS}

Ten rf-plasma MBE grown GaN samples were characterized optically and electrically by a variety of methods. The PPC effect was found to have no connection with any of the parameters investigated, including the magnitude of the yellow luminescence as has been suggested. We have found evidence of at least two different lifetimes involved in PPC and have proposed a possible connection with Ga vacancies. Spectral PC measurements showed several states in the band gap and support the assertion of the role of the Ga vacancy in PPC.

\section{ACKNOWLEDGEMENTS}

We would like to thank Dr. N.C. Giles for allowing M. Moldovan to perform the PL measurements in her laboratory, and for providing some of the equipment used in the PC measurements. This work was supported by ONR Grant N00014-96-1-1008 and monitored by Colin E. C. Wood.

\section{REFERENCES}

1 C.H. Qiu and J.I. Pankove, Appl. Phys. Lett 70, 1983 (1997).

2 C.H. Qiu et al., Appl. Phys. Lett. 66, 2712 (1995).

3 J.I. Pankove et al., Appl. Phys. Lett. 74, 416 (1999).

4 J.Z. Li et al., Appl. Phys. Lett. 69, 1474 (1996)

5 M.T. Hirsch et al., Appl. Phys. Lett. 71, 1098 (1997).

6 G. Beadie et al., Appl. Phys. Lett. 71, 1092 (1997).

7 C. Johnson et al. Appl. Phys. Lett. 68, 1808 (1996).

8 C.V. Reddy et al., Appl. Phys. Lett. 73, 244 (1998).

9 T.H. Myers et al., J. Vac. Sci. Technol. B 17, 1654(1999).

10 A.K. Viswanath et al., J. Appl. Phys. 84, 3848 (1998).

11 H.M. Chen et al., J. Appl. Phys. 82, 899 (1997).

12 U. Kaufmann et al., Phys. Rev. B 59, 5561 (1999).

13 J. Neugebauer and C.G. Van de Walle, Appl. Phys. Lett. 69, 503 (1996).

14 E. Calleja et al.,, Phys. Rev. B 55, 4689 (1997).

15 C.G. Van de Walle, Phys. Rev. B56, 56 (1997). 